\title{
Correction to: Parental Phubbing and Adolescents' Depressive Symptoms: Self-Esteem and Perceived Social Support as Moderators
}

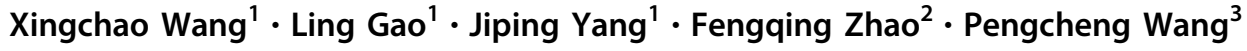

Published online: 5 June 2020

(c) Springer Science+Business Media, LLC, part of Springer Nature 2020

Correction to: Journal of Youth and Adolescence (2020) 49:427-437

https://doi.org/10.1007/s10964-019-01185-x

The original version of the article was inadvertently published with the errors in the values of Tables 1 and 2. The corrections are listed below:

(1) Table 1: skewness of parental phubbing should be 0.43 rather than 0.3 .

(2) Table 1: kurtosis of depressive symptoms should be 2.34 rather than 20.4 .
(3) Table 1: $p<0.01$ rather than $p<0.1$.

(4) Table 2: in step 2, the effect of perceived economic stress on depressive symptoms should be 0.06 rather than 0.6 .

(5) Table 2: in step 3, the effect of perceived social support on depressive symptoms should be -0.05 rather than -0.5 .

(6) Table 2: in step 3, the interaction effect of parental phubbing and perceived social support should be -0.03 rather than -0.3 .

The original article can be found online at https://doi.org/10.1007/ s10964-019-01185-x.

Fengqing Zhao

susanfair@163.com

1 School of Educational Science, Shanxi University, No. 92 Wucheng Road, Xiaodian District, Taiyuan 030006, China

2 School of Education, Zhengzhou University, No. 100 Science Street, Zhengzhou 450001, China

3 The Center of Internet and Social Psychology, Department of Psychology, Renmin University of China, No. 59 Zhongguancun Street, Haidian District, Beijing 100872, China 